\title{
7. FLARE-SURGES
}

R. G. GIOVANELLI

It has been known for twenty years that surges are sometimes ejected from active regions on the Sun during the progress of flares. They appear as regions of strong $\mathrm{H} \alpha$ absorption when viewed on the solar disk, Doppler displacements indicating that matter is ejected at speeds of the order of $100 \mathrm{~km} / \mathrm{sec}$, some at least of the ejected material subsequently falling back to the chromosphere [r].

Flare patrol films obtained with the Sydney $\mathrm{H} \alpha$ monochromatic telescope have been used for studying the general features of these events [2]. Despite reduction in contrast due to Doppler shifts, dark surges have been detected in $18 \%$ of over 400 flares. No statistically significant dependence on flare size has been found, an interesting result since $95 \%$ of the flares are of class I- , of area below IOO $\times \mathrm{IO}^{-6}$ hemisphere, $80 \%$ being less than $50 \times 10^{-6}$ hemisphere. Nor have any dark surges been detected other than those accompanying flares, though these may not have been so readily detected. However, we may conclude that all, or nearly all, dark $\mathrm{H} \alpha$ surges are associated with flares, most of them of class $\mathrm{I}-$.

Three general features of dark $\mathrm{H} \alpha$ surges emerge from these observations:

(i) Most surges overlap or start almost in contact with their associated flares. The surge does not always move radially away from the centre of the flare, but may even be tangential to the flare. However, this conclusion establishes the essential unity of the flare-surge event.

(ii) In disk events well away from the limb, the initial paths of surges appear to lie more or less radially away from the nearest large sunspot. The directions of these surges in elevation are unknown, but the observations nevertheless suggest that the direction of motion is strongly influenced by the magnetic field of the sunspot.

(iii) In $60 \%$ of flare-surges studied on the disk, and in over $80 \%$ of events involving flares of apparent area exceeding $28 \times 10^{-6}$ hemisphere, the dark surge is preceded by a diffuse, usually asymmetric, expansion of the flare. As the diffuse bright material fades, it gives place to a dark surge which then moves outwards. The failure to observe this bright expansion in all flare-surges is probably due to lack of resolution.

It has been possible to study the transition from bright diffuse expansion of the flare to dark surge using a few favourable events. The best was that of 1956 May 18 , in which the flare began at $\mathrm{OI}^{\mathrm{h}} 23^{\mathrm{m}} 52^{\mathrm{B}}$. A sudden bright expansion commenced at $\mathrm{OI}^{\mathrm{h}} 29^{\mathrm{m}} 22^{\mathrm{s}}$. A small faint spike appeared beyond the limb about $9^{\mathrm{m}}$ later, though it had no visible association with the flare at this stage. The first traces of the dark surge were noted simultaneously, in the position formerly occupied by the bright flare expansion; the surge moved out to join the limb spike, also taking about $9-\mathrm{IO}^{\mathrm{m}}$ to do so. These observations indicate clearly that the bright expansion of the flare represented the first stages in the emission of a particle stream, which was eventually faintly visible beyond the limb while still invisible and transparent on the disk. Because of the constancy of the stream velocity, and hence of any Doppler displacements, the subsequent development of the dark surge must have been due to an increase in the opacity of the stream. This general behaviour can be deduced also from several other events.

To interpret its changing appearance, we must recognize that the stream of particles from the flare is initially highly ionized and, moreover, that it expands after ejection. Now, if the temperature increases or remains constant during expansion, the further increase in ionization must produce an increase in transparency in $\mathrm{H} \alpha$, such as is indeed observed in the initial stages; and this may also happen, depending on circumstances, even if the temperature drops. But an increase in absorption, as during the development of the dark surge, can occur only if the temperature decreases.

However, there is not always a decrease in temperature during ejection. For example, there are a few observations of ejected flares, at least one of which increased in brightness during ejection [3]. Theoretically, this can occur only under rather restricted conditions (which is presumably why such events are rare), but an increase in temperature is essential. Thus in some streams there is evidence of heating after ejection. 


\title{
JOINT DISCUSSION
}

\author{
REFERENCES
}

[I] The Sun (edited by Kuiper, G. P.), p. 385: University of Chicago Press, 1953.

[2] Giovanelli, R. G. and McCabe, Marie K. Aust. J. Phys. II, I9I, $195^{8}$.

[3] Bray, R. J., Loughhead, R. E., Burgess, V. R. and McCabe, Marie K. Aust. J. Phys. ro, 319, 1957.

\section{HYDROGEN AND HELIUM EXCITATION IN THE CHROMO- SPHERE AND CHROMOSPHERIC FLARES}

\section{A. KRAT AND V. M. SOBOLEV}

Spectroscopic investigations show that the nature of the excitation of hydrogen and helium atoms in flares is different in different flares. For instance, the appearance of helium lines (more frequently in absorption) is not necessarily accompanied even by comparatively bright flares of importance 2. Observations made at Pulkovo during 1956-8 show that the absorption in $\mathrm{D}_{3}$ usually does not appear in the region of maximal $\mathrm{H} \alpha$-brightness. In this respect observations of spectra of limb flares are of special interest. On I958 April 23 we obtained spectra of a limb flare in a comparatively wide spectral region, which showed the lines $\mathrm{He} 4472$ and $47 \mathrm{r}_{3}$ in emission. We were not able to detect any traces of $\mathrm{He}^{+} 4686$ (Zirin [x]). This can be explained if we assume that the hydrogen lines and those of neutral and ionized helium appear under different physical conditions in different parts of the flare.

V. Krat and L. Pravduke [2] and [3] found that the chromosphere may be considered as consisting of a number of short-lived filaments with temperatures and densities varying within wide limits. We also assume that the chromospheric flares are composed of different filaments or layers with different temperatures and densities. Our observations do not show any evidence of a line broadening of the Balmer series from $\mathrm{H} \delta$ to $\mathrm{H}_{10}$ and $\mathrm{H}_{11}$ due to the Stark effect. There is also no noticeable self-reversal in these lines. However the hydrogen line profiles show that above the flare there is matter causing light scattering. This matter gives rise to absorption line profiles which are narrower than those of the emission lines of the flare and not broader than the ordinary chromospheric lines. Also the ejections from the flare, predominantly directed towards the photosphere, are observed in emission in the lines of hydrogen and ionized calcium $\mathrm{H}$ and $\mathrm{K}$ [4]. Investigation of the problem of helium equilibrium in flares, taking into account the photo-ionization from the level $2^{3} \mathrm{~S}$ and the impacts of the second type for the level $2^{3} \mathrm{~S}$ and other factors, permitted us to calculate the number of $\mathrm{He}$ atoms in various states and to evaluate the ionization of $\mathrm{He}$. As the transitions between the higher levels, for low electron concentration $n_{e}$, are due mainly to photospheric radiation, the ratios of the line intensities $4472 / \mathrm{D}_{3}, 4713 / \mathrm{D}_{3}, 3889 / \mathrm{D}_{3}$ are practically constant for all $T_{e}$, being equal to: $4472 / \mathrm{D}_{3}=0 \cdot 10 ; 47 \mathrm{I} 3 / \mathrm{D}_{3}=0^{\circ} \mathrm{OI} ; 3889 / \mathrm{D}_{3}=0 \cdot 09$.

The normal ratio of the intensities of the lines He 5876,4472,4713,3889 in flares can change only for high electron concentrations, not less than $\mathrm{IO}^{13}$. This points to an extremely high density of the 'helium' filaments of the flare observed by Zirin [x]. In the majority of flares the density of these filaments must be lower. This in general does not contradict the results of E. Mustel and A. Severny [5]. The exceptionally rare appearance of the line $\mathrm{He}^{+}$ $\lambda 4686$ in prominences and flares shows that it cannot be caused either by coronal radiation or by the ultra-violet radiation of the 'helium' filaments themselves. It appears only in subcoronal filaments, which can be formed during the transformation of chromospheric matter into coronal gas or vice versa.

\section{REFERENCES}

[1] Zirin, H. Ap.J. 126, I59, I957.

[2] Krat, V. A. and Sobolev, V. M. Pulkovo Bull. I60, 1957

[3] Krat, V. A. and Pravduke, L. M. Pulkovo Bull. 156, 1956.

[4] Krat, V. A. and Sobolev, V. M. Pulkovo Bull. 162, 1958.

[5] Mustel, E. R. and Severny,A. B. Publ. Crim.astrophys.Obs. 5, 3, 1950;8, 19, 1952 ; 13, 82, r955. 